\title{
The Development of A New, Rapid, Amperometric Immunosensor for the Detection of Low Concentrations of Bacteria Part I: Design of the Detection System and Applications
}

\author{
Eric Carnes and Ebtisam Wilkins \\ Department of Chemical and Nuclear Engineering \\ University of New Mexico, Albuquerque, New Mexico, 87131
}

\begin{abstract}
A portable, flow-through amperometric immunoassay system utilizing a newly designed immunosensor has been developed for the detection of bacteria. A sandwich scheme of immunoassay involving two stages of immunoreaction was employed using Escherichia coli as a model. E. coli antibodies were immobilized on Toray carbon paper to create a disposable immunofiltration membrane. Detection of the bacteria was achieved through the use of horseradish peroxidase-labeled antibodies, which, when coupled with a substrate of hydrogen peroxide and sodium iodide, yielded an electro-reduction, on the order of microamps, which was measured amperometrically. The immunosensor is highly sensitive and selective through the use of the disposable immunofiltration membranes that also function as immunoelectrodes, which enhance the rate of the immunoreaction due to the high surface to volume ratio of the solid to liquid phases. Potential commercialization through easier sample preparation and higher reproducibility of this system was a major objective, so the design highlights simplicity along with functionality. Preliminary data shows concentrations as low as 50 cell $/ \mathrm{mL}$ can be detected in $22 \mathrm{~min}$. Bacteria can be detected but not quantified over the desired working range of 50-1000 cell/mL. Optimization of the system parameters must be completed in order to ensure correct functionality of the sensor over the desired range of detection.
\end{abstract}

Key words: Carbon Paper, Rapid, E. coli, Portable, Immunofiltration, Sensitive

\section{INTRODUCTION}

Fast methods for detection of bacterial contamination are of great interest. For instance, the determination of Salmonella by amperometric detection was performed using a homogenous conjugate of antibodies to Salmonella with alkaline phosphatase enzyme labels. The immunocomplex formed after the interaction with the test sample was separated by filtration through a polycarbonate membrane. The enzyme activity was measured using differential pulse voltammetry. The detection limit was $5 \times 10^{3}$ cell/mL [1]. Another sensor that utilizes lateral flow and capillary action to detect Salmonella and E. coli can detect concentrations as low as $79 \mathrm{CFU} / \mathrm{mL}$ [2]. Systems have also been designed that utilize screen-printed sensor arrays [3] or solid polymer electrolytes [4] in conjunction with amperometric detection.

A piezoelectric immunosensor was developed for the rapid diagnosis of $M$. tuberculosis. A piezoelectric quartz crystal obeys the Sauerbrey equation which describes the relationship between the resonant frequency of a crystal and mass deposited on its electrodes [5]. Tuberculosis was detected by comparing the changes between control and experimental groups and diagnoses were based on the detection of the M. tuberculosis antigen. Tuberculosis was added to a detection cell with a crystal coated in protein $\mathrm{A}$ and anti-TB. It was found that the working range of detection for this system was $10^{6}-10^{8}$ cell $/ \mathrm{mL}$. Another piezoelectric immunosensor was developed for the detection of the hepatitis A antigen. The quartz crystal surface was treated by a glow discharge using ethylenediamine for the replacement of amino groups on the surface of the crystal and then activated by glutaraldehyde to immobilize an antibody. It was found that modified crystals could be used for longer periods of time than standard immobilization [6]. Another sensor has been used to detect Salmonella typhimurium as low as 500,000 CFU/mL [7].

Analysis of contaminated food can also be performed using piezoelectric sensors. The anodic polymerization of o-aminophenol, o-phenylenediamine and $\mathrm{m}$ enlyenediamine was applied to the immobilization of antibodies for the detection of Salmonella paratyphi. The analytical concentration was measured in the range of $10^{5}-10^{9}$ cell $/ \mathrm{mL}[8]$.

The advantage to piezoelectric immunosensors is the possibility of directly monitoring a broad range of analytes in an immunoreaction without labels or reagents. The possibility of preparing highly specific antibodies and simpler techniques are also beneficial factors. A disadvantage is the non-specific binding of the components of a reaction medium to the surface of the crystal, which will lead to low reliability and assay reproducibility. 
The possibility of using one template for the determination of a wide variety of substances is proposed by using a sandwich immunoassay where specific antibodies were immobilized on the surface of a planar waveguide. After the formation of the immunocomplex by the specific antigen, secondary fluorescent-labeled antibodies were added to the solution. The immunoassay was applied to the detection of staphylococcal enterotoxin B, F1 antigen from Yersinia pestis and D-dimer, which is a marker of sepsis and thrombotic disorders. The detection limit is similar to standard ELISA techniques. Yet, the method can also be used for routine analyses because the assay time was shortened from $4-6 \mathrm{~h}$ to $14 \mathrm{~min}$ [9].

The determination of cocaine with alkaline phosphatase labelled polyclonal antibodies was investigated. First, the test sample was incubated with antibodies followed by the separation of unbound antibodies from the immunocomplex on an affinity column. The immunocomplex of cocaine with labelled antibodies was collected and the cocaine concentration was found by the spectrophotometric measurement of the enzyme activity using p-nitrophenyl phosphate as a substrate [10].

There are also many other novel types of types of optical sensors. One sensor uses a resonant mirror to attempt to watch specific reactions as they occur by analyzing the refractive index and thickness at the surface of the mirror [11]. Another sensor involves the use of disposable transparent polymer vials to change the color of a sample solution. This color change can then be optically measured, with Listeria being measured to $1000 \mathrm{CFU}$ over 10-13 h [12]. Different pathogens can also be detected using ion mobility spectrometry in a process that takes $10-20 \mathrm{~h}$ [13]. A few other optical sensors use surface plasmon resonance [14] to assay for bacteria and other contaminants in foods, such milk, chicken and beef [15-17].

Some advantages of optical immunosensors over electrochemical immunosensors are the insensitivity to electrical interfaces and the high safety of in-vivo studies. However, the use of optical immunosensors is less advantageous as compared with other techniques because time consuming, multi-step assays are often required and large, expensive equipment is often needed, which can prevent an assay from being used in the field [18].

Each immunosensor is designed and optimized to function under particular conditions that relate to a specific problem. Various requirements are needed such as sensitivity, rapidity, efficiency and simplicity of assay procedures. A major problem is the reproducibility of determination results depending on the technique and analytical range.

In general, electrochemical sensors are advantageous over other systems because they can operate in turbid media, offer comparable instrument sensitivity and are more amenable to miniaturization. In addition, the continuous response of an electrode system allows for computer control, making the electrochemical detection simpler, more reliable and less expensive than other analytical techniques. Optical immunosensors are usually characterized by low detection limits and lower determination errors over an entire analytical range. Yet, these immunosensors exhibit a narrow analytical range and require expensive and sophisticated instruments, disabling them from field use. This makes them less common than amperometric immunosensors, which tend to be inexpensive and simple to use. However, amperometric immunosensors cannot monitor an immunoreactio directly and use complex systems for signal amplification may be required. In addition, there is a wide range of determination errors depending on the label and immunoassay format. Piezoelectric immunosensors impose strict requirements on the specificity of antibodies because cross-reactions cause significant interfaces. When nonspecific binding is taken into account, the determination errors are relatively low.

A particular type of immunosensor should be chosen depending on the specific analytical problem to be solved, as each sensor has its own advantages. Immunosensors remain of interest to researchers because they have the possibility of miniaturization, shortened analysis times, simplified analytical procedures, lower detection limits and increased assay sensitivities. As a result, progress in the development of new immunosensors depends on an effort made by engineers, analytical chemists, medical personnel, biochemists and ecologists. This study seeks to explore the key features in the design of a new amperometric immunosensor that would allow for rapid and sensitive detection in a system that can be automated or miniaturized with the potential for commercialization. The design utilizes new disposable immunofiltration membranes to simplify the assay procedure while also reducing the cost.

\section{MATERIALS AND METHODS}

Materials: Carbon paper was a courtesy of Toray Composites, Inc. (Tacoma, WA). Carbon rods used as electrodes and current collectors were a courtesy of DFI Pultruded Composites Inc. (Erlanger, KY). Silver used for reference electrodes was from Thunderbird Supply (Albuquerque, NM). Woodward's reagent K (N-ethyl5-phenyliso-xazolium-3'-sulfonate), trypsin inhibitor, sodium chloride, sodium iodide, hydrogen peroxide and Tween 20 were obtained from Sigma Chemical Company (St.Louis, MO). All chemicals used were of analytical grade. Affinity purified antibody to total E.coli and peroxidase-labeled affinity purified antibody to total E.coli (conjugate) were obtained from Virostat (Portland, MA). Distilled water was used for the preparation of all aqueous solutions. 
Preparation of Immunofiltration Membranes: Preparation of the immunofiltration membranes was adapted from a procedure designed for ULTI carbon particles [19]. To first activate the carbon paper, a 20 $\mathrm{mg} / \mathrm{mL}$ solution of Woodward's Reagent $\mathrm{K}$ is prepared in water. Next, discs of Toray carbon paper, 7/8-inch in diameter, (TGP-H-1.0t, pore size 1-5 $\mu \mathrm{m}$ ) are placed in separate glass bottles and immersed in $1 \mathrm{~mL}$ of the above solution. The discs are then incubated while being shaken for $2 \mathrm{~h}$ at room temperature. Following the incubation, the discs are washed 3 times, for $3 \mathrm{~min}$ each time, with $1 \mathrm{~mL}$ of PBS ( $\mathrm{pH}$ 7.8). Then the discs are transferred to a polystyrene plate and allowed to dry. Next, $200 \mu \mathrm{L}$ of an antibody solution $(0.8 \mathrm{mg} / \mathrm{mL})$ is added to each well and allowed to incubate for $8 \mathrm{~h}$ at $4^{\circ} \mathrm{C}$. Finally, each disc is incubated while being shaken for $2 \mathrm{~h}$ in $1 \mathrm{~mL}$ of a solution of $5 \mathrm{mg} / \mathrm{mL}$ of trypsin inhibitor (as a blocking agent) in PBS (pH 7.8). The discs are again washed as described above and stored in $1 \mathrm{~mL}$ of PBS (pH 7.8) at $4^{\circ} \mathrm{C}$ until use.

ELISA Procedure: An ELISA plate is a polystyrene plate with 96 small wells each of capacity $200 \mu \mathrm{L}$. they are sold commercially after pretreatment to adsorb antibodies or antigens. $100 \mu \mathrm{L}$ each of a solution of E.coli antibodies $(4 \mathrm{mg} / \mathrm{mL})$ in PBS $(\mathrm{pH} 7.8)$ was placed into the wells of the ELISA plate and stored overnight at $4{ }^{\circ} \mathrm{C}$. Each well in was then washed three times with PBS ( $\mathrm{pH}$ 7.8). The ELISA plate was then incubated with different concentrations of E.coli in PBS. The plate was incubated at $37^{\circ} \mathrm{C}$ for $2 \mathrm{~h}$. The wells were then washed three times with PBST $(\mathrm{pH}$ 7.8). Peroxidase labeled conjugate was prepared by diluting the commercially obtained solution $(4 \mathrm{mg} / \mathrm{mL})$ to a ratio of 1:1000 in PBS (pH 7.8) and then $100 \mu \mathrm{L}$ of the diluted immunoconjugate solution was added into each of the wells. This was followed by incubation of the plate at $37^{\circ} \mathrm{C}$ for $2 \mathrm{~h}$. Later, the wells were washed three times with PBST (pH 7.8). Then $100 \mu \mathrm{L}$ of TMB substrate, consisting of hydrogen peroxide and tetramethylbenzidine, was added to each well and produced an observable color change. The absorbance of each well is then read by an ELISA plate reader at a wavelength of $450 \mathrm{~nm}$. This was used to generate a calibration curve. For subsequent blind samples, the same procedure was followed, only the blind samples, including blanks, were used instead of the known concentrations of $E$. coli in buffer [20].

Immunoassay Procedure: The reaction of E. coli and its antibodies has been studied at length [21]. The following immunoassay procedure was used to obtain preliminary data and determine the feasibility of this new immunosensor design and modified to use a flow rate of $200 \mu \mathrm{L} / \mathrm{min}[22]$ :

* Pre-washing stage: flow of a rinsing buffer solution, PBST ( $\mathrm{pH} 7.8$ ), for a duration of $2 \mathrm{~min}$.
* First stage of immunoreaction: flow analyte in PBS (pH 7.8) for a duration of $2 \mathrm{~min}$.

* First washing stage: flow of PBST (pH 7.8) alone for a duration of $2 \mathrm{~min}$.

* Second stage of immunoreaction: flow of immunoconjugate $(0.08 \mathrm{mg} / \mathrm{mL})$ in PBS $(\mathrm{pH} 7.8)$ for a duration of $9 \mathrm{~min}$.

* Second washing stage: flow of PBST (pH 5.6) for a duration of $2 \mathrm{~min}$.

* Amperometric measurement stage: flow of substrate $(0.1 \mathrm{mM})$ in PBS $(\mathrm{pH} 5.6)$ for $5 \mathrm{~min}$. Amperometric output of iodine formed as a result of the enzymatic oxidation of iodide is recorded under a $105 \mathrm{mV}$ bias.

\section{RESULTS AND DISCUSSION}

Immunoassay System Design: Conventional immunoassay techniques, such as ELISA, suffer from several critical limitations. The first major limitation is caused by the complexity of the techniques. Preparation of the various reagents are often timeconsuming and laborious [23]. Then, the tests performed can be quite rigorous or require bulky, expensive, specialized equipment [24]. This results in a technique that not only is quite difficult to automate, but also requires highly trained technicians to operate, driving costs up even higher. Another limitation is caused by the requirement for analyte diffusion from the bulk solution to the solid support on which the antibodies are immobilized [25, 26]. Due to these diffusion limitations, the time required in order to achieve equilibrium in the reaction between immobilized antibodies and the antigen in solution is usually on the order of several min to several $\mathrm{h}$. A third major limitation results from the low ratio of immunologically reactive surface to the volume of the liquid reaction medium. This small ratio greatly compromises the efficiency of antibody-antigen interactions, limiting the sensitivity of the assay. Yet another limitation is the required separation of bound and unbound immunoagents, which is usually done by conducting various washing stages. This separation is often laborious and difficult to automate, prolonging the analysis time.

There are several approaches to overcome these limitations. One could attempt to design a system that was simple to prepare as well as to operate, in order to allow for automation of the assay, along with reducing the need for highly trained operators. This would greatly reduce the cost of the immunoassay. Another approach would be to reduce the transport limitations across the liquid boundary layer surrounding the solid phase support, which would serve to increase the system's efficiency while also decreasing the assay time. One could also increase the antibody binding capacity of the immunologically reactive surface, or increase the ratio of the solid support surface to the 
liquid phase, in order to increase the sensitivity of the assay. An effective design of an immunoassay system aims at encompassing all of the above approaches and hence achieving improved analytical characteristics of enzyme-linked heterogeneous immunoassays.

Flow-through System: The use of a flow-based system aims at minimizing the transport limitation of conventional immunoassays by actively bringing the immunoagents in contact with the solid support. This eliminates the diffusion limitations that result from the use of stationary media. This also results in enhanced interaction between the immunoagents, hence improving the sensitivity of the assay. The use of a flow-based system also allows for easier automation of the assay, since the flow would be driven by pumps and valves that are easily controlled by today's computer technology. Automation of the system would lead to more cost-effective assays, as well as more reliable results. The problem with the necessity of washing stages is also addressed in a flow-based system, where flow of a rinsing solution can simply be added whenever desired.

The flow-through design is based on a continuous flow concept. An important advantage of continuous flow is the ability to continuously pump solutions until a steady state is reached. Steady-state operation permits easy and precise measurement and is suitable for use when ease and precision of signal measurement is required and when the reagents are inexpensive or used in small amounts. Steady-state operation also allows the phenomena occurring within the system to be investigated using steady-state approximations.

Amperometric Detection: The use of carbon paper as an immunofiltration membrane lends itself well to amperometric detection, since the membrane also functions as an immunoelectrode (the working electrode). Amperometric detection of enzyme-labeled immunoagents is desirable because it allows for signal amplification, due to the high activity of enzymes, resulting in an enhancement of the sensitivity of the assay. Amperometric detection also allows for miniaturization and portability of the immunoassay system, since the equipment that would be required is readily available. A sandwich-scheme can be utilized, which is illustrated in Fig. 1. In this scheme, antibodies specific to the analyte are immobilized on the surface of the carbon paper to form the immunofiltration membrane. The analyte is then passed through the immunofiltration membrane, where they become entrapped by the immobilized antibodies. A second set of antibodies (immunoconjugate), which are labeled and can therefore be detected, is then passed through the system and allowed to bind to the analyte which has been captured.
Three-electrode System: There are various advantages to using a three-electrode in certain systems where high sensitivity and accuracy are needed [27]. Two electrode systems consist of a reference electrode and a working electrode. A potential is applied to the electrochemical cell and the current is then recorded as a function of this potential. However, some disadvantages appear in this system. As the reference electrode carries current, the electrode will polarize, resulting in an overpotential. This leads to an unknown potential at the working electrode, leading to inaccurate measurements in sensitive systems. Another disadvantage of the two-electrode system is the inaccuracy caused by consumption of the reference electrode. These problems can be overcome by using very large reference electrodes, which is not a viable option when portability or miniaturization of the system is desired. The best solution to these problems comes in the use of a three-electrode system.

In a three-electrode system, besides the working and reference electrodes, a counter electrode is introduced. This gives a true reference electrode for controlling the potential and a counter electrode for current injection. This results in a system which gives much more accurate results when operating at high sensitivity levels.

Commercially Available Hardware and Reagents: The use of commercially available products contributes to the overall simplicity of the system, as well as helping to maintain cost effectiveness. Commercially available immunoreagents are of very high quality due to the sophisticated purification methods employed. Due to this, though, they tend to have a relatively high price. However, due to the extreme complexity involved in obtaining such high quality products, inhouse production of immunoagents would be more expensive and would probably yield a decreased quality of product, which could compromise the function of the immunoassay.

Utilizing commercially available hardware, like pumps, lines and other components, decreases the overall system cost and simplifies future manufacturing, automating and miniaturizing procedures. Another benefit of using commercially available products is the enhanced reliability. Commercially available immunoagents and chemicals are tested to maintain purity and hardware components can easily be repaired or replaced should they require maintenance or fail to function. This helps to keep the system simple and cost effective, while still allowing for a reliable and highly sensitive immunoassay.

Immunofiltration Membranes: The use of an immunofiltration membrane serves to localize where the immunoreactions take place, enhancing the intimate 


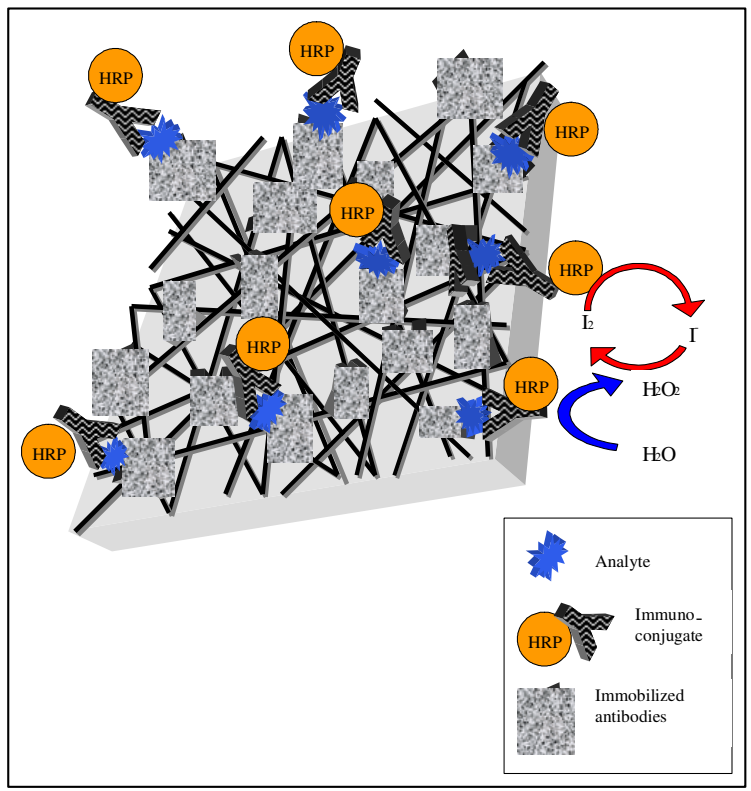

Fig. 1: Representation of the Sandwich Scheme on the Immunofiltration Membrane Utilized in the Amperometric Detection

contact required for the immunoagents to interact and facilitating the concentration of the analyte from relatively large sample volumes of a dilute concentration. A membrane constructed of a porous substance would increase the ratio of reactive surface area to the volume of the liquid media in which the immunoagents are present. Use of an immunosorbent substance would allow for more antibodies to be immobilized on the surface, increasing the reactivity of the surface and thereby increasing the sensitivity of the assay.

An immunofiltration membrane also functions well in a flow-through system, allowing for the construction of an inexpensive, disposable solid support. This would simplify the assay procedure and reduce its cost. A main drawback of most heterogeneous flow-systems is the need for regeneration of the antibody-modified solid support after each assay, requiring the use of chaiotropic reagents to break the antigen-antibody bond. This results in a loss of enzyme activity as well as increasing the cost and complexity of the assay [28]. The use of an immunofiltration membrane permits construction of elements that are disposable. This would ensure constant activity of the immobilized immunoagents and eliminate the need for regeneration of the support, which would lead to simpler and more reliable operation. It also permits easy adaptation of the immunoassay system to the detection of a wide range of analytes, simply depending of the type of antibody immobilized on the surface of the immunofiltration membrane.

A design based on the use of [29] carbon paper as the solid support for the immunofiltration membrane is proposed. The paper is micro-porous, as can be seen in Fig. 2 and the carbon is both immunosorbent and conductive. The carbon paper offers a large surface area to volume ratio and, at the same time, functions as the working electrode because of its conductive nature. The carbon paper consists of pores whose size is on the same order of magnitude as the target analytes, making it ideal for filtration. The carbon itself possesses characteristic pores whose size is of the same order as

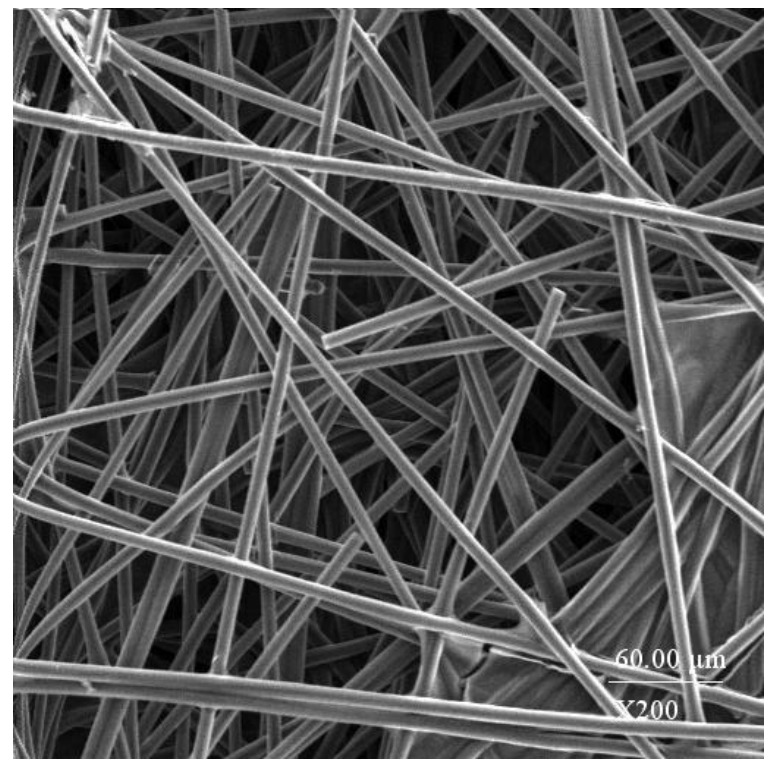

Fig. 2: SEM Microphotograph of Toray Carbon Paper most antibodies, making it also ideal as an immunosorbent.

The use of carbon paper directly as an immunofiltration membrane eliminates the problems associated with 
construction of a complex disposable sensing element used in previous flow-through immunosensors [30].Disposable sensing elements have previously consisted of plastic columns with membrane filters on which the immunosorbent is deposited by centrifugation in order to form an immunoelectrode to be used in electrochemical detection. This requires extensive preparation in order to create, driving up the cost of the assay and increasing possibilities for error. However, the use of carbon paper results in an immunofiltration membrane that is also the working immunoelectrode. This keeps the system as simple as possible, while also reducing cost and increasing the sensitivity and reliability of the assay.

Immunosensor Design: The design of this new immunosensor is based on the utilization of a flowthrough technique in conjunction with an immunofiltration membrane that also serves as an immunoelectrode [28]. A cutaway view of the design is shown in Fig. 3. The design serves to house the disposable immunofiltration membrane and provide optimum conditions for the immunoreaction. The sensor is constructed of silastic and Teflon plastic to ensure that none of the immunoagents absorb into the sensor casing, which could lead to unreliable measurements. The sensor is also designed to allow for a range of flow rates, while maintaining the greatest possible area for interaction of the various immunoagents at the immunofiltration membrane surface in order to give a high sensitivity. This also

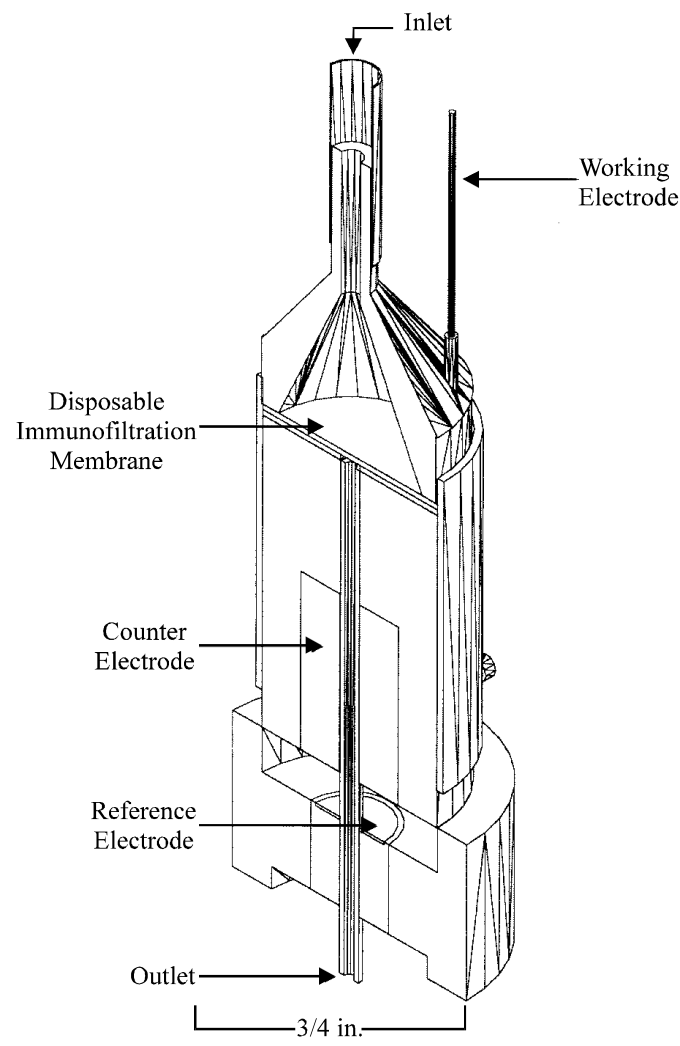

Fig. 3: Cut Away View of Immunosensor Design Prototype

eliminates a limitation of most conventional flowthrough immunosensors, which usually have been limited to very low flow rates that lead directly to longer analysis times.

Flow enters the sensor through the top, then goes through a dispersion head, which is similar to a shower head and is allowed to accumulate in the chamber that houses the immunosensor. This serves to maximize the volume of immunoagents that come in contact with the immunofiltration membrane in order to increase the conversion of the immunoreaction. The chamber is quickly filled and steady-state operation is established. Flow continues down through a carbon counter electrode and then through an $\mathrm{Ag} / \mathrm{AgCl}$ reference electrode before finally exiting the system. A current collector, constructed of carbon, is connected to the immunofiltration membrane to create a functional immunoelectrode. This working electrode, combined with the previously mentioned counter and reference electrodes, effectively collects the amperometric output of the sensor.

The optimization of the sensor design should be noted. The sensor functions as a plug-flow reactor that has been packed with a catalyst, which can be thought of as the immunofiltration membrane. A plug-flow reactor is the optimum reactor design to use when the concentration of the reactants being used is low and a

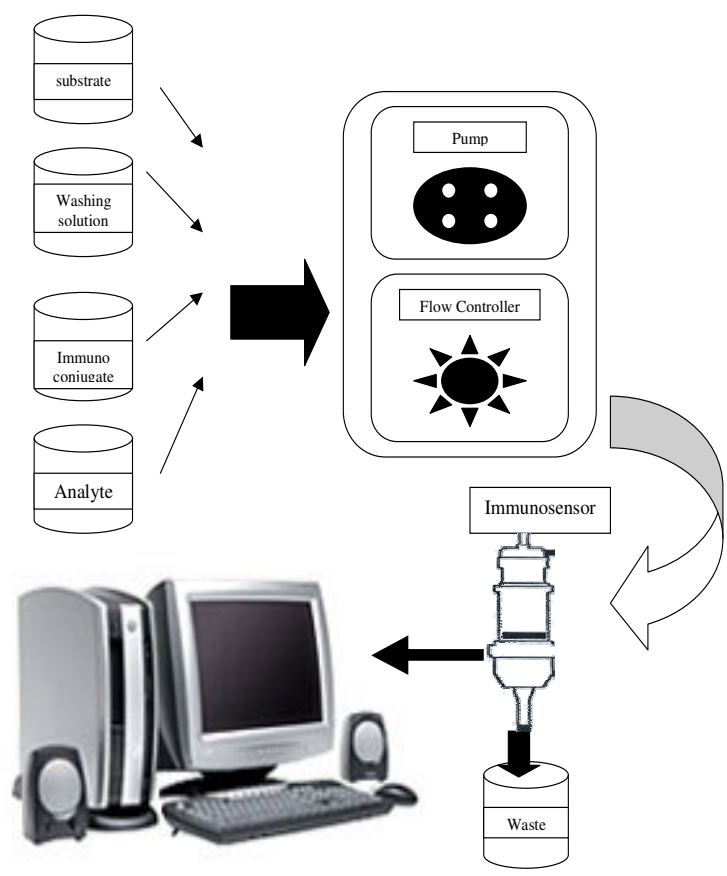

Fig. 4: Schematic of Flow-through Immunoassay Lab Set-up

first order reaction mechanism is assumed [31]. Since the immunoreactions that happen on the immunofiltration membrane are relatively fast, the reactions become limited by diffusion of the reactants to the surface of the membrane. The small size of the sensor maintains a laminar flow (the maximum flow rate of pump used in this study gives a Reynolds number of 0.95), so direct analysis of the diffusion can be done. Despite the laminar flow, proper contact of the analyte with the surface of the immunofiltration 
membrane is ensured by the size and layout of the fibers comprising the membrane. It is found that the continuous flow of reagents can react at the surface of the immunofiltration membrane very effectively due to a large surface area to volume ratio of $309 \mathrm{~cm}^{2} / \mathrm{cm}^{3}$ (data provided by manufacturer [29]). The simplicity of the design and use of readily available hardware also contribute to the immunosensor design. The counter electrode is made of carbon, which is inexpensive but also immunosorbent. After many uses, the counter electrode can begin to absorb a small amount of the immunoagents, which could compromise the functionality of the immunosensor. However, since carbon is readily available, the counter electrode can simply be replaced after a time, before it becomes saturated with immunoagents. Also, the reference electrode is constructed of $\mathrm{Ag} / \mathrm{AgCl}$, which is available quite inexpensively from various medical device suppliers. This results in a reference electrode that is not only simple and inexpensive, but that is also effective and that should not react with the immunoagents or different matrices in which those

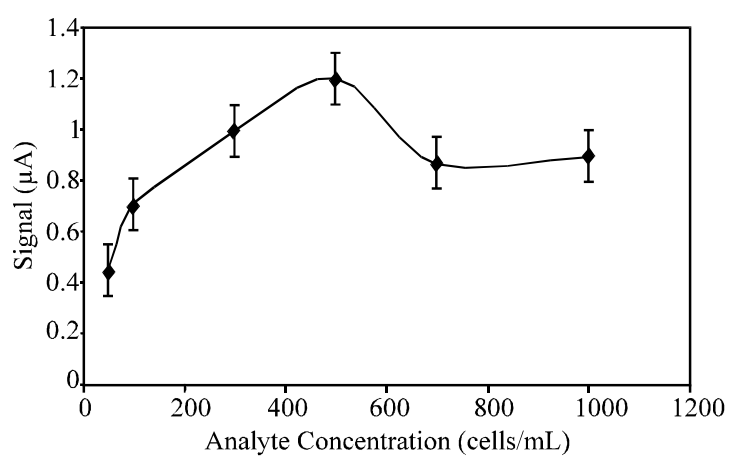

Fig. 5: Calibration Curve for E. coli Using Optimized Immunoassay Procedure Showing Linear Dependence on Concentration Over Working Range (50-1000 cell/mL)

agents are suspended. The use of these readily available electrodes, combined with the disposable immunofiltration membrane of Toray carbon paper and a casing constructed out of available plastics, yields a sensor that is simple and inexpensive, as well as being efficient, reliable and highly functional.

Immunoassay System Layout: The schematic of the flow-through immunoassay system is shown in Fig. 4. The simple system consists of the immunosensor, a peristaltic pump with a variable-rate flow controller, reagent and waste vessels and an electrochemical/data recorder interface. The immunosensor is connected to the pump using silastic tubing that was designed to release any air bubbles presented in the line. Flow of the different reagents through the pump could easily be controlled by a 5-way valve to allow for future automation of the system. A variable-rate pump has been used to optimize the flow rate of the system, but in future manufacturing, a single speed pump operating at the optimum flow rate would be used to minimize cost and maintain steady-state operation. The electrochemical interface is used to provide the working potential for the immunosensor and to process the output signal. The entire system has been construction out of components that are readily available in order to maintain simplicity and reliability, as well as facilitating commercialization.

Preliminary Data: The immunoassay procedure listed earlier was used to test the design discussed above to ensure its feasibility to detect low concentrations of bacterial pathogens. Total E. coli was used as model. From Fig. 5, it can be seen that bacteria were detected in a range of $50-1000$ cell $/ \mathrm{mL}$. This is thought to be a desirable working range because it covers most government mandated regulations for bacterial contamination. However, due to the non-linearity of the data, the number of bacteria present in the sample cannot be quantified. The trend appears

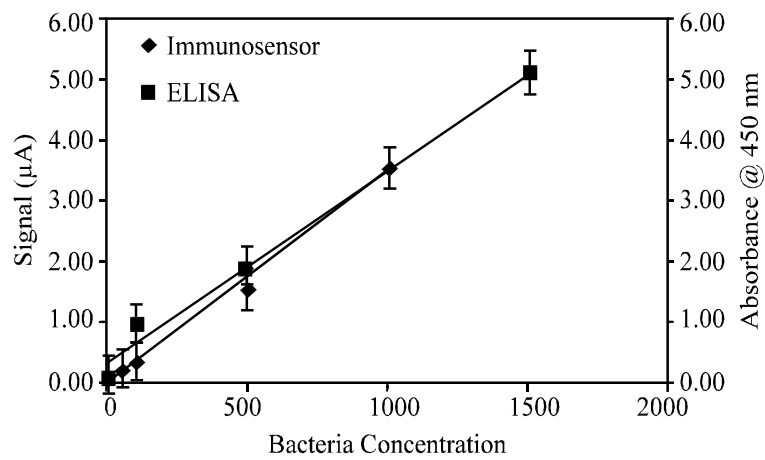

Fig. 6: Calibration Curves Using E. coli in Human Plasma

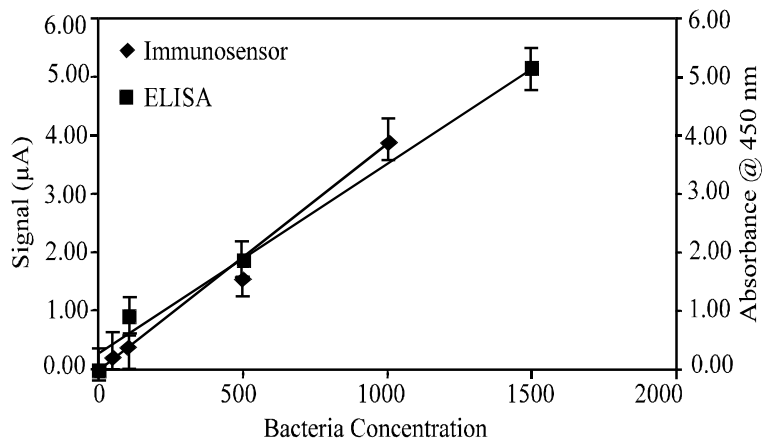

Fig. 7: Calibration Curves Using E. coli in Saliva

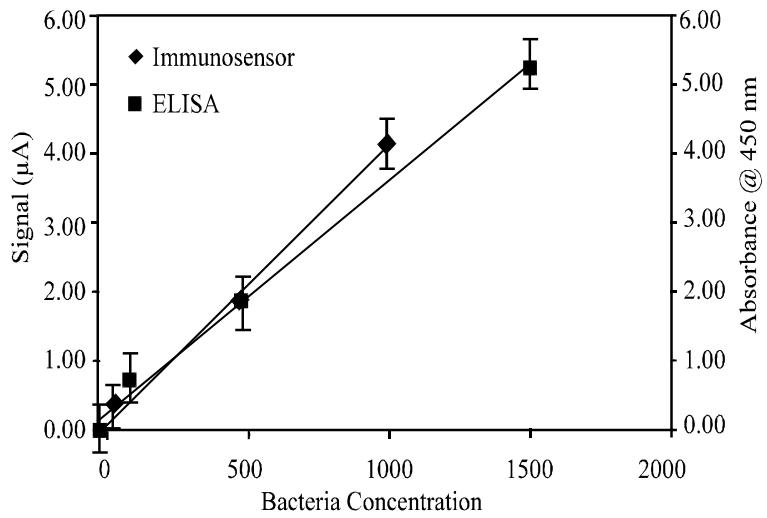

Fig. 8: Calibration Curves Using E. coli in Dialysate 
to show a saturation of the sensor response with increasing analyte concentration. Optimization of the immunoassay parameters must be conducted in order to be able to effectively quantify bacteria over the desired working range.

Applications of the Immunosensor System

Blood Infections: In potentially fatal neonatal meningitis, E. coli will begin to colonize in the blood stream. In order for rapid detection of bacteria in blood, which could lead to fewer infant fatalities, the new immunosensor system was calibrated using human plasma free of bacteria, obtained from United Blood Services. The plasma was then diluted with water in a 1:1 ratio and passed through a $53 \mu \mathrm{m}$ filter to make it fluid enough for use in the sensor. The plasma was then spiked with concentrations of 50, 100, 500 and 1000 cell $/ \mathrm{mL}$ and a calibration curve for the sensor and the ELISA was generated and shown in Fig. 6. At low bacteria concentrations, it can be seen that the linear regression of the ELISA does tend to zero, despite the fact that the ELISA reader calibrates itself by subtracting the absorbance of a blank sample of media (containing no bacteria), in this case plasma, from each other sample. Hence, when the data is fit linearly, zero absorbance should correspond to zero bacteria. However, this is not the case, due to the fact that the ELISA is not accurate at low concentrations. Even with the most modern substrates, the ELISA is only reliable down to 100 cell $/ \mathrm{mL}$ of $E$. coli. The immunosensor, though, maintains a linear trend towards zero when the current of the blank has been subtracted. This shows that the immunosensor system can accurately detect very low concentrations of bacteria in human plasma samples.

Respiratory Infections: Although concentrations of bacteria in saliva can range a great deal from unavoidable variables, such as one's diet, it would be beneficial to be able to test for bacteria in saliva in conjunction with the many respiratory infections that can occur due to bacteria, similar to the throat-swab, culture test for Streptococcus that most people are probably familiar with. Since it would be beneficial to be able to rapidly detect these bacteria and allow for proper treatment to begin before complete infection has set in, the immunosensor system was tested using bacteria-free human saliva diluted with water in a 1:2 ratio in order to make it fluid enough for use in the sensor. The saliva was then spiked with known concentrations of bacteria over a range of 50 to 1000 cell $/ \mathrm{mL}$ and used with the sensor, as well as the ELISA, to generate calibration curves, which are shown in Fig. 7. As with the blood samples, the immunosensor data compares quite well with the standard technique and even exceeds performance at low concentrations. Due to the high sensitivity and short time needed to perform this assay, as opposed to the two days normally required for a strep-throat culture, it seems that this new immunosensor system may have the potential to aid in the detection of infections of the respiratory system caused by bacteria.

Peritonitis: To evaluate the immunosensor performance in real samples for use in other medical applications, samples of dialysate were evaluated to determine if $E$. coli was present, which could lead to peritonitis. First, sterile dialysate was spiked with known concentrations of bacteria to generate a calibration curve for the sensor, as well as the ELISA, which is shown below in Fig. 8. Next, blind samples that were known to have bacterial contamination were assayed using the newly developed sensor and the

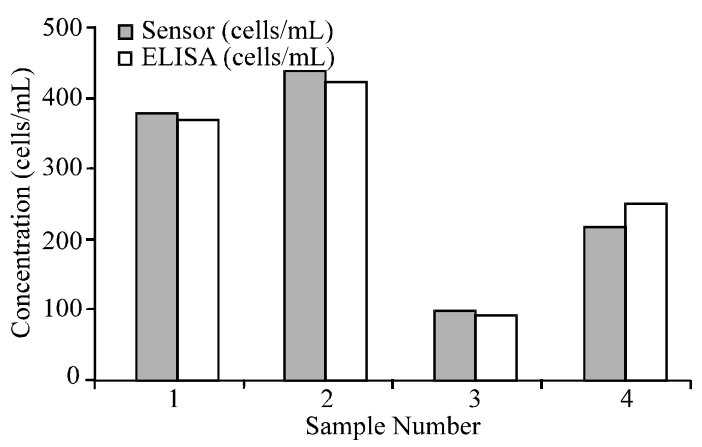

Fig. 9: Comparison of New Immunosensor to Standard ELISA on Blind Peritonitis Samples

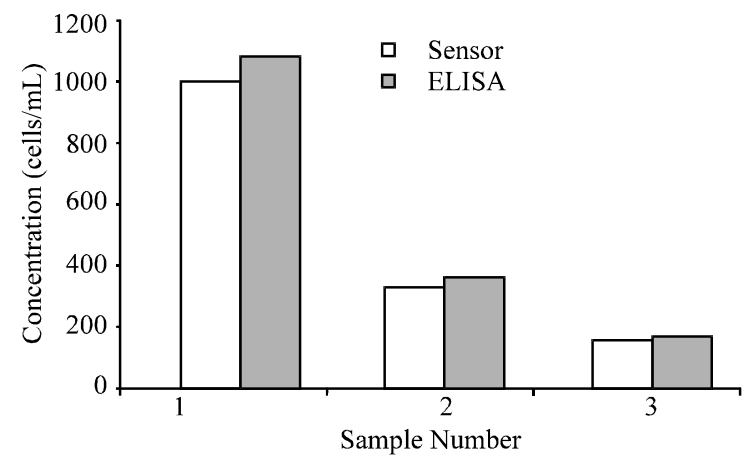

Fig. 10: Comparison of New Immunosensor to Standard ELISA on Chlorinated Effluent Samples

results were compared to that of a standard ELISA, shown in Fig. 9. It can be seen that the new sensor system gives results that are with $20 \%$ of the standard ELISA values. However, the immunosensor quantified the bacteria in $17 \mathrm{~min}$, while preparation and use of the ELISA took two full days. Also, a much smaller volume of immunochemicals can be utilized by the sensor, which can lead to significant cost decrease if many samples are being tested. This means that the sensor can be a good alternative to the ELISA and has the potential to be used in similar medical applications.

Waste Water: Water treatment facilities all over the world must monitor the amount of bacteria and other contaminates present during various stages of the treatment. To demonstrate the feasibility of this new immunosensor system to detect $E$. coli in waste water samples, samples of chlorinated effluent were obtained 
from the City of Albuquerque Water Treatment Facility. The effluent was estimated to contain $10^{4}$ cell $/ \mathrm{mL}$ of bacteria from previous data obtained at the facility. The sample was then diluted 10, 50 and 100 times and tested on the immunosensor. The number of bacteria in the samples was quantified using the standard sensor calibration curve in buffer, since blank water yields a background current similar to buffer. The diluted effluent samples were also tested using a standard ELISA and the results are presented below in Fig. 10. The sensor is able to quantify the bacteria within $10 \%$ of the standard detection method, while providing results much quicker and using a much smaller volume of reagents. From these results, it can be seen that the sensor is capable of detecting bacteria in environmental applications, such as waste water treatment.

\section{CONCLUSION}

A new, rapid, amperometric immunoassay system has been developed and its feasibility has been demonstrated on analyte samples of total E. coli. The design is comprised of a new immunosensor which utilizes disposable immunofiltration membranes that also function as immunoelectrodes in order to overcome the limitations of conventional assays. The immunoassay should be able to be easily adapted for the analysis of a wide range of analytes by changing the antibodies that are used in the assay. The immunoassay system was also designed to be readily commercialized through automation and miniaturization. A portable, automated prototype is currently under construction.

A sandwich scheme assay has been utilized, with HRPlabeled antibodies being used in the amperometric detection. Sodium iodide and hydrogen peroxide form the substrate of the peroxidase enzyme to produce iodine by the enzymatic oxidation of the iodide ions. The amperometric signal produced by the electroreduction of the iodine at the immunoelectrode was directly proportional to the amount of analyte present in a sample solution. Amperometric detection was chosen due to many benefits, including its inherent ability to be used in miniaturized, portable systems. The disposable immunofiltration membrane, the main element of this new design, was constructed from Toray carbon paper. $E$. coli antibodies were then immobilized on the surface of the carbon paper using Woodward's Reagent K immobilization to form the disposable immunofiltration membranes. Since the membranes are comprised of conductive carbon, they also function as an immunoelectrode. These immunofiltration membranes serve to increase the local concentration of agents in the immunoreaction, resulting in higher sensitivity of the assay. The fact that they are simple and disposable, as well as being highly effective, translates into more feasible commercialization of this immunoassay system than any comparable model.
Another novel feature of this immunoassay design is the flow-through immunosensor. The sensor has been designed to function simply and effectively. It can handle a wide range of flow rates, something that previous sensors could not normally do. Inexpensive and easily replaceable materials were used for the electrodes in the sensor. The rest of the sensor is constructed from commercially available pieces, allowing it to be massed-produced quite efficiently while still maintaining the effectiveness of the original design. The few hardware components of the system were also commercially available and consist of a pump with a flow controller, potentiostat and multi-meters.

Preliminary data showed that concentrations as low as 50 cell $/ \mathrm{mL}$ of $E$. coli could be detected in $22 \mathrm{~min}$. However, the data did not allow one to quantify the bacteria over a desired working range of 50-1000 cell $/ \mathrm{mL}$. The design was also shown to be feasible in various medical and environmental applications. Further optimization of the immunoassay parameters must occur in order for the immunoassay system to meet the design objectives.

\section{ACKNOWLEDGEMENTS}

The authors gratefully acknowledge the financial support of this work by the National Science Foundation. Appreciation also goes to the University of New Mexico School of Engineering for additional financial support. The authors would also like to thank Sagar Yelleti, Sireesha Chemburu and Dr. Jasmin Shah for their assistance.

\section{REFERENCES}

1. Yang, Z.P., Y.B. Li, C. Balagtas, M. Slavik and D. Paul, 1998. Immunoelectrochemical assay in combination with homogeneous enzyme-labeled antibody conjugation for rapid detection of Salmonella. Electroanalysis, 10: 913-916.

2. Muhammad-Tahir, Z. and E.C. Alocilja, 2003. A conductometric biosensor for biosecurity. Biosensors and Bioelectronics, 18: 813-819.

3. Ertl, P., M. Wagner and S.T. Mikkelsen, 2003. Rapid identification of viable Escherichia coli subspecies with an electrochemical screen-printed biosensor array. Biosensors and Bioelectronics, 18: 907-916.

4. Opekar, F. and K. Stulike, 1999. Electrochemical sensors with solid polymer electrolytes. Analytica Chimica Acta, 385: 151-162.

5. Fengjiao, H.E. and Liude Zhang, 2002. Rapid diagnosis of M-tuberculosis using a piezoelectric immunosensor. Analytical Sci., 18: 397-401. 
6. Mutlu, S., R. Saber, C. Kocum and E. Piskin, 1999. An immunosensor: Immobilization of anti-HBs antibody on glow-discharge treated piezoelectric quartz crystal for HBs-AG detection. Analytical Lett., 32: 317-334.

7. Ye, J., S.V. Letcher and A.G. Rand, 1997. Piezoelectric biosensor for detection of Salmonella typhimurium. J. Food Sci., 62: 1067.

8. Si, S.H., F.L. Ren, W. Cheng and S.Z. Yao, 1997. Preparation of a piezoelectric immunosensor for the detection of Salmonella paratyphi A by immobilization of antibodies on electropolymerized films. Fresenius J. Analytical Chem., 357: 1101-1105.

9. Rowe, C.A., L.M. Tender, J.P. Felden, S.B. Scruggs, B.D. Maccraith, J.J. Cras and F.S. Ligler, 1999. Array biosensor for simultaneous identification of bacterial, viral and protein analytes. Analytical Chem., 71: 3846.

10. Eremenko, A.V., C.G. Bauer, A. Makower, B. Kanne, H. Baumgarten and F.W. Scheller, 1998. The development of a non-competitive immunoenzymometric assay of cocaine. Analytica Chimica Acta ,358: 5-13.

11. Dmitriev, D.A. and Y.S. Massino et al., 2002. Analysis of the binding of bispecific monoclonal antibodies with immobilized antigens (human IgG and horseradish peroxidase) using a resonant mirror biosensor. J. Immunological Methods, 261: 103-118.

12. Firstenburg-Eden, R. and L.A. Shelef, 2000. A new rapid automated method for the detection of Listeria from environmental swabs and sponges. Intl. J. Food Microb., 56: 231-237.

13. Strachan, N.J.C., F.J. Nicholson and I.D. Ogden, 1995. An automated sampling system using ion mobility spectrometry for the rapid detection of bacteria. Analytica Chimica Acta, 313: 63-67.

14. Koubova, V. and E. Brynda et al., 2001. Detection of foodborne pathogens using surface plasmon resonance biosensors. Sensors and Actuators, 74: 100-105.

15. Wahlstrom, L.,1998. Proceedings of the $25^{\text {th }}$ International Dairy Congress. Dairy Sci. Technol., 2: 344.

16. Hauck, S., C. Kosslinger, S. Drost et al., 1998. Lebensmittelchemie, 52: 158.

17. Carlson, M.A., C. B. Bargeron and R.C. Benson et al., 2000. An automated, handheld biosensor for aflatoxin. Biosensors and Bioelectronics, 14: 841-848.

18. May, M., 1999. Nanotechnology: Thinking small. Environmental Health Perspective 107: A450.
19. Krishnan, R., A.L. Ghindilis, P. Atanasov and E. Wilkins, 1995. Fast amperometric immunoassay utilizing highly dispersed electrode material. Analytical Lett., 28: 2459-2474.

20. Chamburu, S., 2003. An electrochemical biosensor for detection of pathogenic bacteria. M. Sc. Thesis, University of New Mexico.

21. Ghindilis, A.L., R. Krishnan, P. Atanasov and E. Wilkins, 1997. Flow-through amperometric immunosensor: Fast 'sandwich' scheme immunoassay. Biosensors and Bioelectronics, 12: 415-423.

22. Abdel-Hamid, I., D. Ivnitski, P. Atanasov and E. Wilkins, 1999. Flow-through immunofiltration assay system for rapid detection of $E$. coli O157:H7. Biosensors and Bioelectronics, 14: 309316.

23. Ivnitski, D., P. Atanasov, I. Abdel-Hamid and E. Wilkins, 2000. Application of electrochemical biosensor for detection of food pathogenic bacteria (Review). Electroanalysis, 12: 317-325.

24. Vetcha, S., I. Abdel-Hamid, P. Atanasov, D. Ivnitski and E. Wilkins, 2000. Portable immunosensor for the fast amperometric detection of anti-hantavirus antibodies. Electroanalysis, 12: 1034-1038.

25. Valcarcel, M. and M.D. Luque de Castro, 1994. Flow-through biochemical sensors. Part V: Flow through sensors based on integrated reaction, separation and detection. Amsterdam, pp: 259-322.

26. Beyer, K., M. Reinecke, W. Noe and T. Scheper, 1995. Immunobased elution assay for process control. Analytica Chimica Acta, 309: 301-305.

27. Lambrechts, M. and W. Sansen, 1992. Biosensors: Micreelectrochemical devices. Bristol (England), Institute of Physics Publishing.

28. Abdel-Hamid, I., 1999. Development of a portable immunoassay system for rapid and sensitive analyte detection. University of New Mexico Dissertation.

29. Toray Data Sheet, 2003. Toray Composites (America), Inc., $1900250^{\text {th }}$ Ave. E., Tacoma, WA 98446.

30. Vetcha, S., T. Yates, B. Hjelle and E. Wilkins, 2001. Rapid and sensitive handheld biosensor for detection of hantavirus antibodies in wild mouse blood samples under field conditions. Submitted to Talanta, 2001.

31. Fogler, H.S., 1999. Elements of Chemical Reaction Engineering. Prentice-Hall, $3^{\text {rd }}$ Edn. 